\title{
Generation of bisymmetric magnetic fields in galaxies with tidal interaction
}

\author{
A. Vögler ${ }^{1,2}$ and D. Schmitt ${ }^{1,3}$ \\ 1 Universitäts-Sternwarte Göttingen, Geismarlandstr. 11, 37083 Göttingen, Germany \\ 2 Max-Planck-Institut für Aeronomie, Max-Planck-Str. 2, 37191 Katlenburg-Lindau, Germany \\ 3 Copernicus Gesellschaft, Max-Planck-Str. 13, 37191 Katlenburg-Lindau, Germany \\ e-mail: egs@copernicus .org
}

Received 11 August 2000 / Accepted 23 April 2001

\begin{abstract}
Tidal interactions between neighbouring galaxies are expected to induce significant nonaxisymmetric velocities in their disks. It has been suggested that these velocities play an important role in the generation of bisymmetric magnetic fields observed in interacting galaxies. We investigate the effect of a nonaxisymmetric radial outflow on a three-dimensional linear mean field dynamo. We find that the usually dominant axisymmetric quadrupole is effectively damped by the outflow. For sufficiently high velocities a bisymmetric magnetic mode is then preferentially excited. The resulting field has a spiral-arm structure extending well into the differentially rotating outskirts of the disk. The influence of velocity-induced mode-coupling effects on bisymmetric field generation is found to be negligible. While being highly idealized, the model seems to give reasonable representations of the large scale fields of the interacting galaxies M 81 and M 51.
\end{abstract}

Key words. galaxies: magnetic fields; interactions - magnetic fields - MHD

\section{Introduction}

Radio observations indicate that in some galaxies, notably M 81 and M 51, but also M 33 and NGC 2276, the globalscale magnetic field has a nonaxisymmetric component. In M 81 the magnetic field is mainly bisymmetric (Krause et al. 1989), whereas the field of M 51 consists of axisymmetric and bisymmetric components of roughly equal strength in the disk, and a mainly horizontal axisymmetric halo field of opposite direction (Berkhuijsen et al. 1997). In both cases the nonaxisymmetric fields reach into the differentially rotating regions of the disk. There are signs of bisymmetric fields in M 33 (Buczilowski \& Beck 1991) and NGC 2276 (Hummel \& Beck 1995), but here the evidence is still equivocal and new data show no dominating $m=1$ field for M 33 (Beck, priv. comm.).

The explanation of bisymmetric field structures still poses serious problems to galactic dynamo theory. While application of standard mean field dynamo theory predicts dominant axisymmetric fields (Beck et al. 1996), several suggestions have been made to overcome the destructive effect that differential rotation of the galactic disk has on bisymmetric field configurations (Rädler 1986). These include azimutal modulation of the $\alpha$-effect (Moss et al. 1993; Rohde et al. 1999; Schreiber \& Schmitt 2000), long

Send offprint requests to: A. Vögler,

e-mail: avoegler@linmpi.mpg.de lived nonaxisymmetric seed fields (Poezd et al. 1993) and effects resulting from tidal interaction with a neighbouring galaxy (Moss 1995, 1996). This paper focuses on the last of these suggestions. The galaxies mentioned above all show signs of interaction with a close companion, which can give rise to nonaxisymmetric velocity fields in the galactic disk with amplitudes of the order of some $10 \mathrm{~km} \mathrm{~s}^{-1}$ (Thomasson \& Donner 1993).

Previously, several mechanisms have been identified that may enhance the generation of bisymmetric fields in the presence of tidal velocities. These velocities $\boldsymbol{u}$ and the magnetic field $\boldsymbol{B}$ can be written as Fourier sums, $\boldsymbol{u}=\sum \boldsymbol{u}_{m}, \boldsymbol{B}=\sum \boldsymbol{B}_{m}$, with $\boldsymbol{u}_{m}, \boldsymbol{B}_{m} \propto \mathrm{e}^{i m \phi}$. Then a $\boldsymbol{u}_{1}$ velocity component can act on a $m=0$ magnetic field mode and drive a slaved $m=1$ magnetic field by means of the $\boldsymbol{u}_{1} \times \boldsymbol{B}_{0}$ term in the dynamo equation. Secondly, the growth rate of the $\boldsymbol{B}_{1}$ component is directly affected by the $\boldsymbol{u}_{2} \times \boldsymbol{B}_{1}$ interaction (e.g. Moss 1996). In addition, a $\boldsymbol{u}_{0}$ velocity component, which acts on the $m=0$ and $m=1$ magnetic field modes separately without causing mode coupling, might enhance the growth rate of the bisymmetric component relative to the axisymmetric one.

In this paper we investigate the influence of a simple, time independent velocity field on a fully $3 \mathrm{D}$, linear, kinematic dynamo model. The velocity field consists of $m=0$ and $m=2$ components only, thus the last two of the mechanisms mentioned above are included in our calculations. 


\section{The model}

The mean field dynamo equation

$\frac{\partial \boldsymbol{B}}{\partial t}=\boldsymbol{\nabla} \times(\boldsymbol{v} \times \boldsymbol{B}+\alpha \boldsymbol{B}-\eta \boldsymbol{\nabla} \times \boldsymbol{B})$

is solved in three spatial dimensions using cylindrical coordinates $(r, \phi, z)$.

Here $\boldsymbol{v}$ is the total velocity consisting of the galactic rotation $\Omega r \boldsymbol{e}_{\phi}$ and a superimposed tidal velocity field $\boldsymbol{u}$. Figure 1 depicts the rotational profile we use. Near the axis of rotation the velocity increases almost linearly corresponding to nearly rigid rotation. In the outer regions the rotation curve becomes flat with a maximum velocity of $250 \mathrm{~km} \mathrm{~s}^{-1}$. The rotation is assumed independent of height.

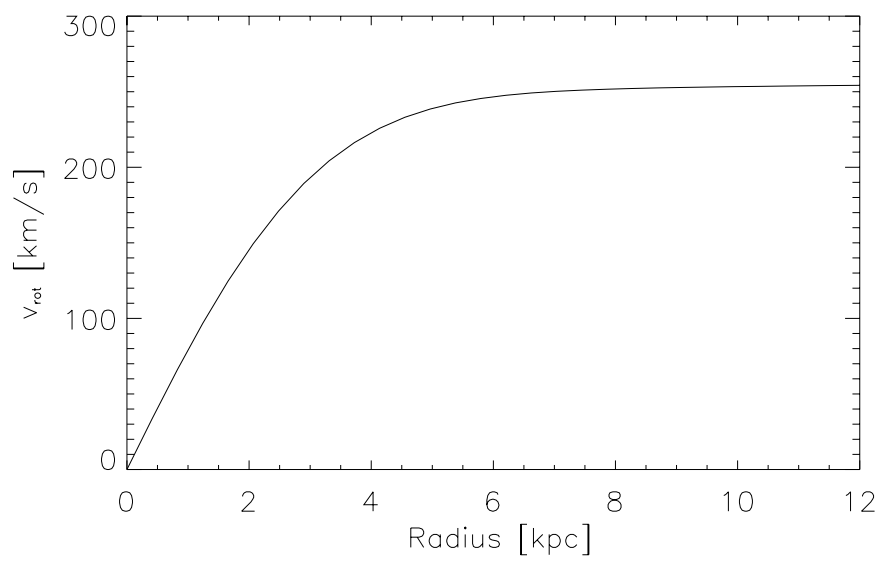

Fig. 1. The rotation curve.

The $\alpha$-tensor is adopted from a model of Rüdiger \& Kitchatinov (1993). We use the expressions

$\alpha_{r r}=\alpha_{\phi \phi}=-\alpha_{0} \frac{4}{15} \tau_{\text {corr }}^{2} \frac{\mathrm{d}<u^{\prime 2}>}{\mathrm{d} z} \Omega$

$\alpha_{z z}=\alpha_{0} \frac{8}{15} \tau_{\text {corr }}^{2} \frac{\mathrm{d}<u^{\prime 2}>}{\mathrm{d} z} \Omega$

with the nondiagonal components set to zero. $\alpha_{0}$ is a common scaling factor. The turbulent diffusivity is given by the scalar expression

$\eta=\frac{1}{3}<u^{\prime 2}>\tau_{\text {corr }}$.

The values we chose for the turbulent velocity $u^{\prime}=$ $<u^{\prime 2}>^{1 / 2}$ and correlation time $\tau_{\text {corr }}$ are based on models in which the turbulence is driven by supernova explosions in the galactic disk. The correlation time is set to $\tau_{\text {corr }}=2.5 \times 10^{7}$ yr. For $u^{\prime}$ we use a profile that decreases from $15 \mathrm{~km} \mathrm{~s}^{-1}$ in the galactic midplane to $8 \mathrm{~km} \mathrm{~s}^{-1}$ in the halo (Schultz et al. 1994).

The tidal velocity field $\boldsymbol{u}$ we used is a simple nonaxisymmetric radial outflow. It has no $z$-dependence and is assumed constant in time. Figure 2 shows a vector plot of the velocity field at the galactic midplane. It consists of a superposition of $m=0$ and $m=2$ Fourier components of equal amplitude. Its influence on magnetic field generation is illustrated by a Fourier decomposition of Eq. (1) (keeping only the term including $\boldsymbol{u}$ on the rhs). This yields

$\frac{\partial}{\partial t} \sum_{m=-M}^{M} \boldsymbol{B}_{m} \mathrm{e}^{i m \phi}=\nabla \times \sum_{m, n=-M}^{M} \boldsymbol{u}_{n} \times \boldsymbol{B}_{m} \mathrm{e}^{i(m+n) \phi}$

( $M=4$ in our calculations), from which

$\frac{\partial}{\partial t} \boldsymbol{B}_{l}=\sum_{m, n=-M}^{M} \boldsymbol{\nabla}_{n+m} \times\left(\boldsymbol{u}_{n} \times \boldsymbol{B}_{m}\right) \delta_{n+m}^{l}$

follows by multiplication with $\exp (-i l \phi)$ and integration over $\phi$. Here $\nabla_{n+m} \times$ is the curl operator with the $\phi$ differentiation already carried out. The particular choice of the tidal velocity field leads to the decoupling of magnetic field modes which are even and odd in $m$. Within a given azimutal symmetry mode mixing occurs due to the $m=2$ component of the tidal velocity field. In the following section the amplitude $u_{\max }$ of $\boldsymbol{u}$ is varied while the overall profile of the velocity field remains unchanged.

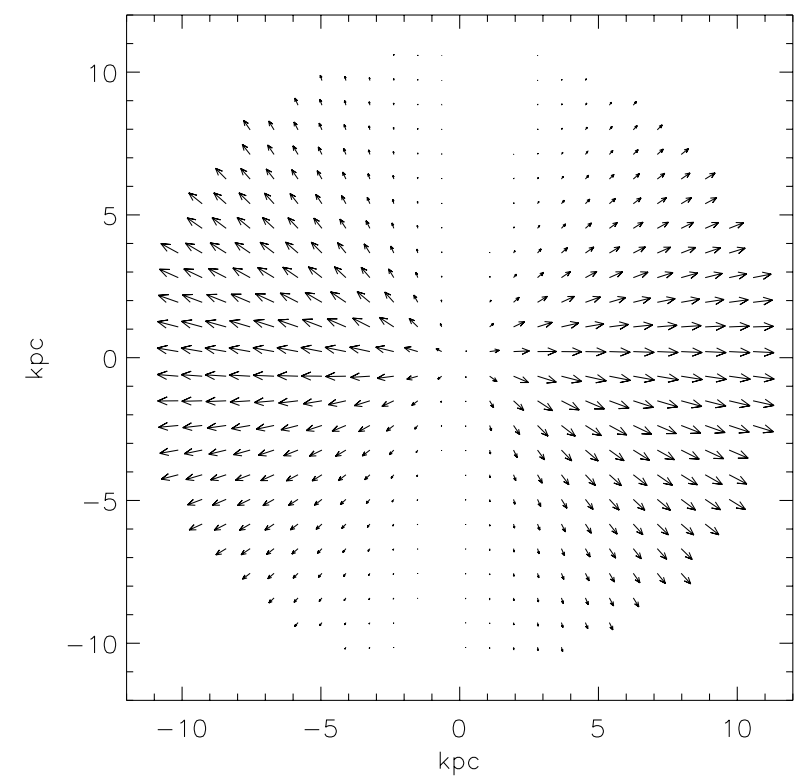

Fig. 2. Vector plot of the tidal velocity field in the galactic midplane.

In all calculations shown in the following section the computational domain extends to $12 \mathrm{kpc}$ in radius and $4 \mathrm{kpc}$ in height. The computations are confined to one half-plane by prescribing symmetric and antisymmetric boundary conditions at the midplane. The treatment of the outer boundary conditions is based on the approach by Elstner et al. (1990). Near the numerical boundaries the turbulent diffusivity is artificially increased, modelling the smooth transition from the galactic disk to a surrounding with vanishing conductivity. Thus the magnetic field is confined to the inner regions of the computational domain and is not affected by the particular choice of 
conditions at the outer boundaries. For convenience, we specified perfect-conductor conditions there.

\section{Results}

First we computed critical values of $\alpha_{0}$, for which the magnetic field is constant or oscillates with constant amplitude, for varying amplitudes of the tidal velocity field; the four magnetic field symmetries that are decoupled (even and odd in $m$, each for symmetric $(\mathrm{S})$ and antisymmetric (A) parity with respect to the midplane) have been investigated separately (Fig. 3). For small values of $u_{\max }$ an even $\mathrm{S}$ mode has by far the smallest critical $\alpha$. We found the mode to be essentially axisymmetric and monotonous (modulated with very weak oscillating $m=2$ and $m=4$ components), so a S0 mode is clearly dominant (see Tab. 1). This is in accordance with previous results for the disk (Elstner et al. 1992) and torus dynamo (Deinzer et al. 1993). As $u_{\max }$ grows, the $\alpha_{\mathrm{c}}$ values increase for all symmetries, indicating that the tidal velocity field has a damping effect on the dynamo. This behaviour is reminiscent of a recent result of Moss et al. (2000), who found that radial flows suppress dynamo action in a thin disk.

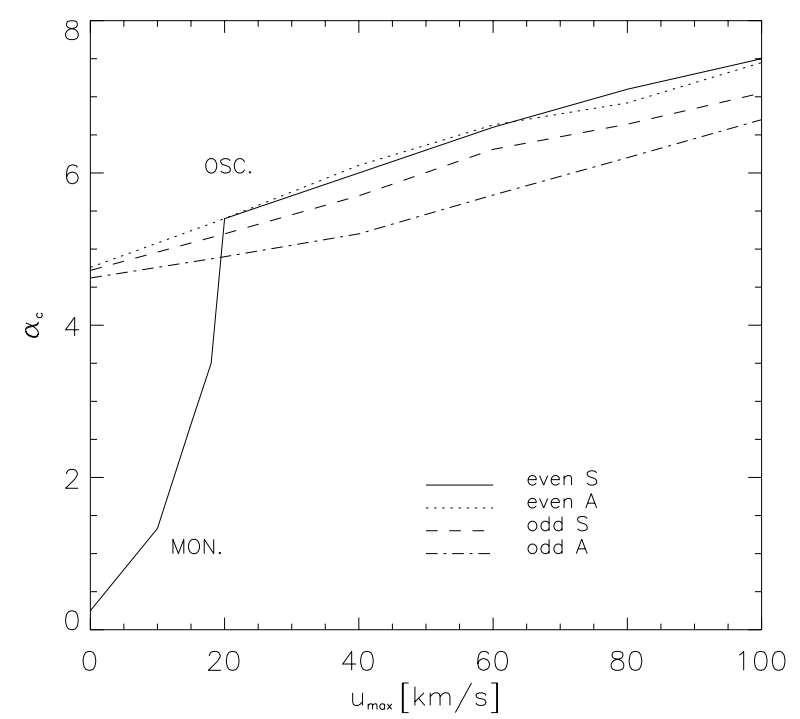

Fig. 3. Critical $\alpha$ as a function of tidal velocity amplitude $u_{\max }$ for the four magnetic field symmetries.

An especially sharp increase in $\alpha_{\mathrm{c}}$ is observed for the S0 mode. Between $u_{\max }=0$ and $u_{\max }=20 \mathrm{~km} \mathrm{~s}^{-1} \alpha_{\mathrm{c}}$ jumps by a factor of about 20 . At $u_{\max } \approx 20 \mathrm{~km} \mathrm{~s}^{-1}$ the mode undergoes a transition from monotonous to oscillatory while remaining predominantly axisymmetric. The solutions for even A and odd (both $\mathrm{S}$ and A) symmetries are oscillatory throughout the whole parameter range shown in Fig. 3. For $u_{\max } \gtrsim 20 \mathrm{~km} \mathrm{~s}^{-1}$ the dominance of $\mathrm{S} 0$ is broken and the odd A mode, which is essentially bisymmetric (see Table 2), is most easily excited. Thus, around this bifurcation point only the stationary S0 and the A1 mode are stable, the oscillatory S0 mode as well
Table 1. Critical $\alpha$, energy ratios and monotonous/oscillatory behaviour in time for the even $\mathrm{S}$ modes.

\begin{tabular}{cccc}
\hline$u_{\max }\left[\mathrm{km} \mathrm{s}^{-1}\right]$ & $\alpha_{\mathrm{c}}$ & $E_{2} / E_{0}$ & Mon./Osc. \\
\hline 0 & 0.25 & - & Mon. \\
10 & 1.3 & $1.4 \times 10^{-3}$ & Mon. \\
15 & 2.7 & $3.4 \times 10^{-3}$ & Mon. \\
20 & 5.4 & $7.0 \times 10^{-3}$ & Osc. \\
40 & 6.0 & $2.2 \times 10^{-2}$ & Osc. \\
80 & 7.1 & $6.0 \times 10^{-2}$ & Osc. \\
\hline
\end{tabular}

Table 2. Critical $\alpha$, energy ratios and monotonous/oscillatory behaviour in time for the odd A modes.

\begin{tabular}{cccc}
\hline$u_{\max }\left[\mathrm{km} \mathrm{s}^{-1}\right]$ & $\alpha_{\mathrm{c}}$ & $E_{3} / E_{1}$ & Mon./Osc. \\
\hline 0 & 4.6 & - & Osc. \\
40 & 5.2 & $9.6 \times 10^{-3}$ & Osc. \\
80 & 6.2 & $6.8 \times 10^{-2}$ & Osc. \\
\hline
\end{tabular}

as all other modes being dominated by the odd A mode for values of $u_{\max }$ larger than $20 \mathrm{~km} \mathrm{~s}^{-1}$.

Further insight into the behaviour of the S0 mode can be gained from Fig. 4. Here, for fixed values of $u_{\max }$ the growth rate $\gamma$ is plotted against the dynamo excitation number $\alpha_{0}$. For $u_{\max }=10 \mathrm{kms}^{-1} \gamma$ first increases for growing $\alpha_{0}$. It reaches a maximum at $\alpha_{0} \approx 4$, then slightly declines before growing steeply for $\alpha_{0} \gtrsim 5$. The critical excitation for $\alpha_{0}=1.3$ corresponds to the monotonous solution. As $u_{\max }$ assumes higher values, the $\gamma\left(\alpha_{0}\right)$ curve is essentially shifted towards lower values of $\gamma$. For $u_{\text {max }} \gtrsim 17 \mathrm{~km} \mathrm{~s}^{-1}$ the minimum at $\alpha_{0} \approx 5$ becomes negative and two additional critical excitation values can be found. So, in the narrow parameter range between $17 \mathrm{~km} \mathrm{~s}^{-1}$ and $20 \mathrm{~km} \mathrm{~s}^{-1}$ three critical values exist simultaneously. For even higher values of $u_{\max }$ the monotonous mode is no longer excited. Two of the critical values disappear, only the one corresponding to the oscillating S0 solution remains.

The non-monotonous dependence of the growth rate $\gamma$ on the parameter $\alpha_{0}$ can be explained by the fact that the steady S0 mode remains excited only throughout a certain range of $\alpha_{0}$, below which the simultaneous action of $\alpha$-effect and large-scale shear is too weak to overcome magnetic diffusion, and above which both mechanisms no longer act in concert towards constructive dynamo action. Parker (1971a, 1971b) was the first who provided a physical interpretation of this phenomenon in the framework of a one-dimensional analytical model. His assertion was subsequently confirmed by numerical simulations of dynamos in thin shells (Schmitt \& Schüssler 1989), in galactic disks (Elstner et al. 1992; Ferrière \& Schmitt 2000), in accretion disks (Torkelsson \& Brandenburg 1994), and in tori (Deinzer et al. 1993). Likewise the transition from steady to oscillatory behaviour occurs when the growth rate of the steady mode is overtaken by the growth rate of the next-order mode. Bifurcations of this kind were previously 
Table 3. Critical $\alpha$ and energy ratios from computations including only the $\boldsymbol{u}_{2}$ velocity component.

\begin{tabular}{cccc}
\hline$u_{\max }\left[\mathrm{km} \mathrm{s}^{-1}\right]$ & Mode & $\alpha_{\mathrm{c}}$ & $E_{2} / E_{0}$ \\
\hline 80 & even S & 0.26 & 0.11 \\
160 & & 0.26 & 0.47 \\
\hline 80 & even A & 4.63 & 0.1 \\
160 & & 4.27 & 0.4 \\
\hline$u_{\max }\left[\mathrm{km} \mathrm{s}^{-1}\right]$ & Mode & $\alpha_{\mathrm{c}}$ & $E_{3} / E_{1}$ \\
\hline 80 & odd S & 4.68 & 0.11 \\
160 & & 4.35 & 0.38 \\
\hline 80 & odd A & 4.56 & 0.07 \\
160 & & 4.43 & 0.27 \\
\hline
\end{tabular}

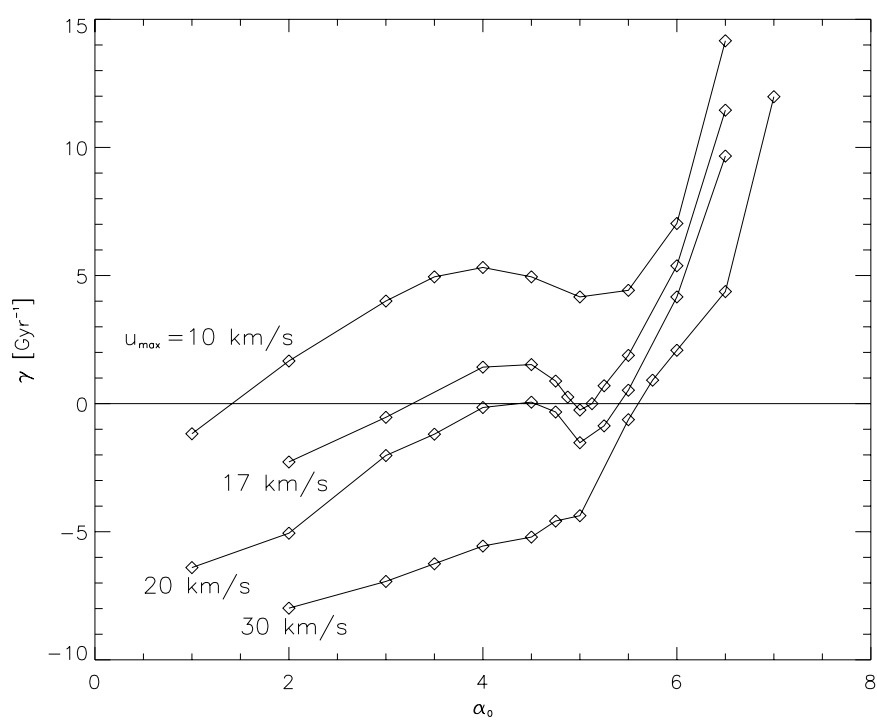

Fig. 4. Growth rates $\gamma$ of the S0 mode as a function of $\alpha_{0}$ for several values of $u_{\max }$.

revealed by the torus dynamo calculations of Deinzer et al. (1993).

We then investigated, how far the above results depend on mode-coupling effects. For that purpose we made computations in which we dropped the $\boldsymbol{u}_{0}$ component which does not contribute to mode coupling and retained solely the $\boldsymbol{u}_{2}$ part of the tidal velocity field. The results, given in Table 3, are quite different from that for the full $\boldsymbol{u}$ field.

While mode coupling readily feeds energy into the higher slaved modes, the critical $\alpha$ values are only slightly altered, even for velocities of more than $100 \mathrm{~km} \mathrm{~s}^{-1}$. There is a tendency towards lower $\alpha_{\mathrm{c}}$ values with increasing velocity amplitude, the dominance of the S0 mode however remains unbroken.

On the other hand, in calculations that included only the axisymmetric $\boldsymbol{u}_{0}$ component, dropping $\boldsymbol{u}_{2}$, we were able to reproduce qualitatively the behaviour of the dynamo for the full $\boldsymbol{u}_{0}+\boldsymbol{u}_{2}$ velocity field. Especially the steep increase in critical $\alpha$ for S0, which results in a dominant A1 mode (the transition occurring here at a velocity amplitude of $u_{0, \max } \sim 10 \mathrm{~km} \mathrm{~s}^{-1}$ ), is recovered. So
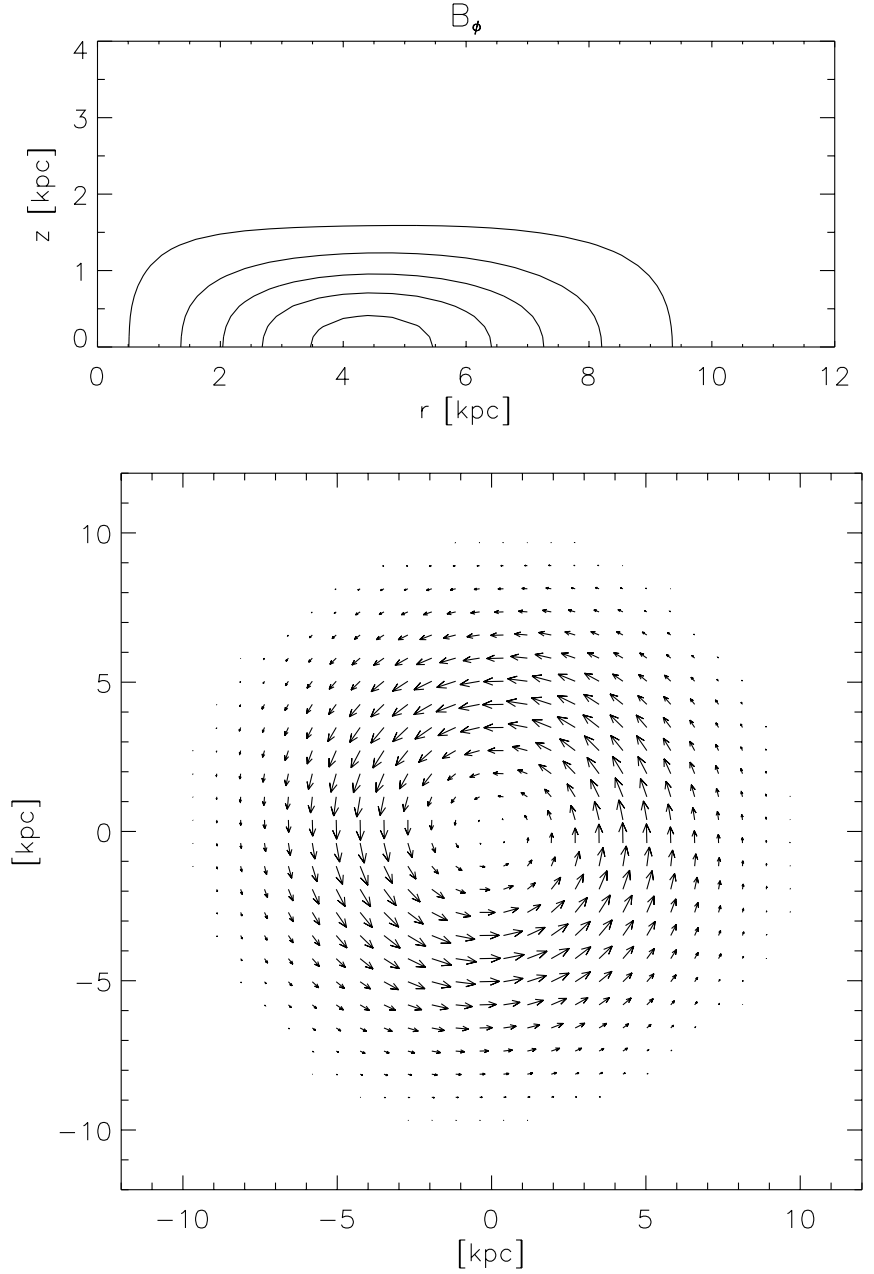

Fig. 5. The field of the monotonous S0 mode for $u_{\max }=0$ : contour plot of the $\phi$-component (top) and field vectors in the galactic midplane (below).

the dynamo behavior appears to be dominated by the axisymmetric outflow while mode coupling has virtually no influence on the excitation properties of the magnetic field.

Finally we present some magnetic field configurations from calculations with the full velocity field. Figure 5 shows the monotonous S0 mode. The mainly toroidal field has a ring-like shape concentrated around a radius of $r \approx 4.5 \mathrm{kpc}$ and is uniformly orientated throughout the whole computational domain. In contrast, the oscillatory modes undergo a characteristic reversal of field direction with height above the galactic midplane. As an example the field of the oscillating even S mode is shown in Fig. 6 . Here two ring-like structures appear, one in the equatorial plane, another one with opposite field orientation $800 \mathrm{pc}$ above.

Vector plots of the odd A field are shown in Fig. 7 . In the absence of a tidal outflow $\left(u_{\max }=0\right)$ the field is concentrated around the rotational axis where rotation is nearly rigid. As the tidal velocity increases the field extends more and more into the differentially rotating regions of the disk and assumes a characteristic spiral shape. 

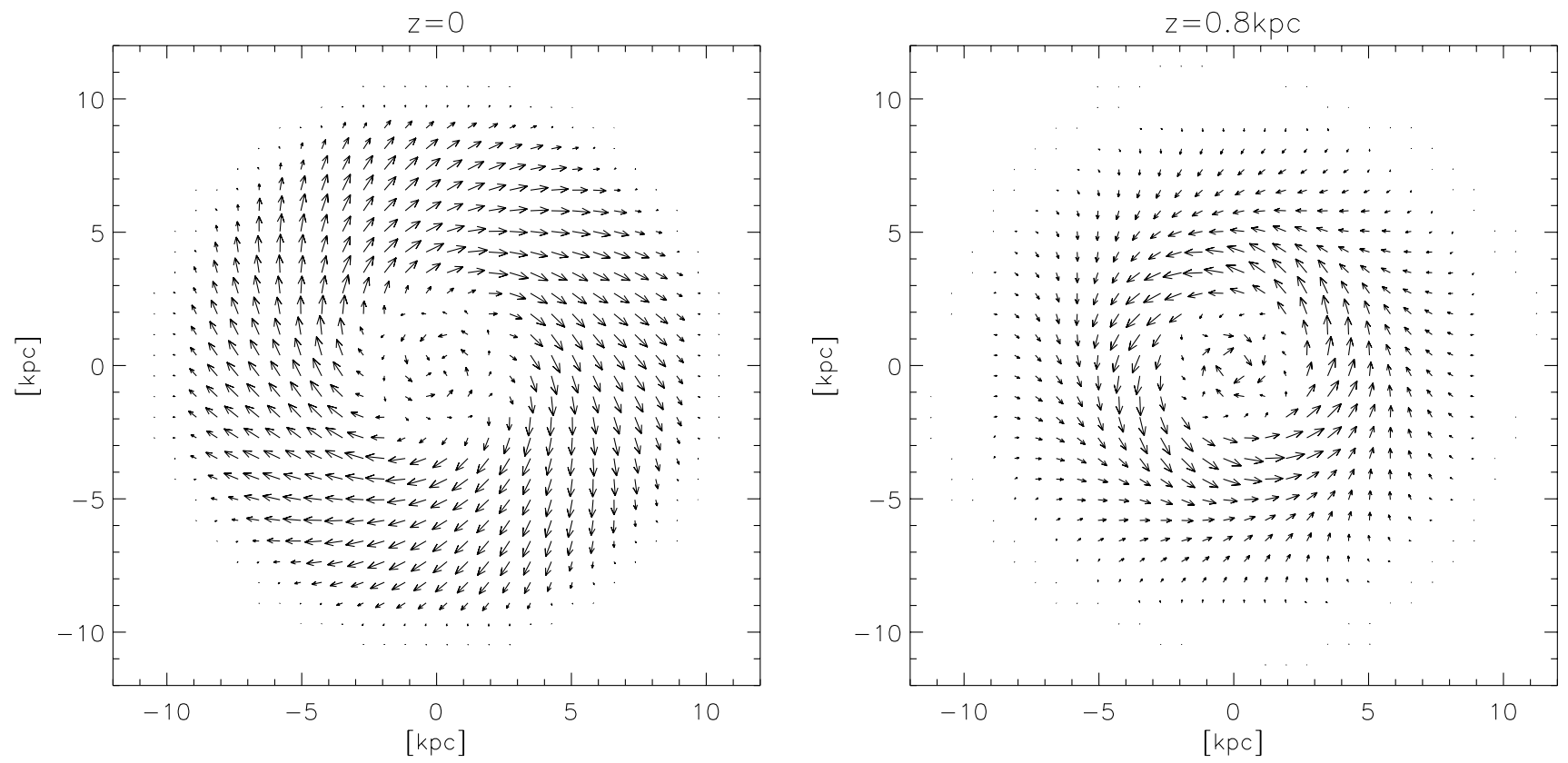

Fig. 6. Magnetic field vectors of the oscillating even $\mathrm{S}$ mode $\left(u_{\max }=60 \mathrm{~km} \mathrm{~s}^{-1}\right)$ in the midplane (left) and $0.8 \mathrm{kpc}$ above (right).
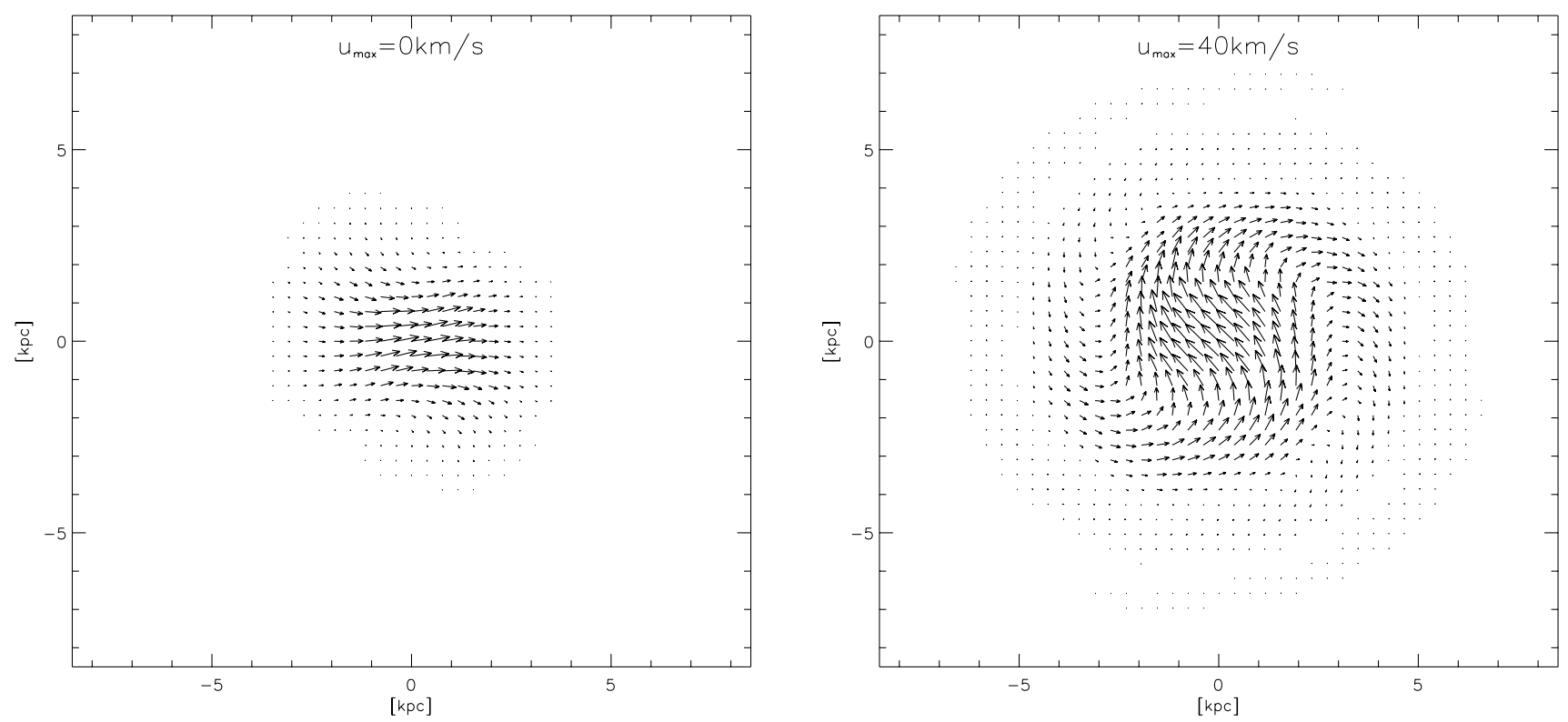

Fig. 7. Magnetic field vectors of the odd A mode for two different tidal velocity amplitudes. Only the inner part of the computational domain is shown.

\section{Summary and discussion}

The aim of this study was to examine the influence of nonrotational large scale velocities, which are expected to result from tidal interaction, on the excitation properties of the galactic dynamo. For small velocity amplitudes we find the monotonous S0 mode to be clearly dominant over all other modes, a result that is well known for dynamos in flat, quasi one-dimensional geometries. For higher modes to become excited the dominance of the steady S0 mode has to be broken. In our study this is achieved by imposing a radial outflow on the disk, but it has also been observed in thicker disks or shells (Hoyng et al. 1994), for larger correlation times $\tau_{\text {corr }}$ of the turbulence (Elstner et al. 1996), or for enhanced diffusion in spiral arms (Schreiber \& Schmitt 2000). Then a number of usually oscillatory modes, symmetric and antisymmetric with respect to the equator, both axisymmetric and nonaxisymmetric, have similar excitation conditions and a variety of field configurations can be obtained, the preferred one depending on the details of the model. In our model a A1 magnetic mode with a spiral-arm structure becomes preferentially excited for sufficiently strong velocity amplitudes. As a result of field transport due to the outflow the spiral 
extends into the differentially rotating regions of the disk, as is observed in M 81 (Krause et al. 1989). All oscillatory modes undergo a field reversal with height, which, in the case of the oscillating S0 mode, results in a field configuration reminiscent of recent observations in M 51 (Berkhuijsen et al. 1997). It must however be kept in mind that the oscillating S0 mode cannot compete with the A1 mode except very close to the bifurcation point at $u_{\text {max }} \approx 17 \mathrm{~km} \mathrm{~s}^{-1}$.

While it is tempting to relate our results to observed fields in galaxies like M 81, it must be emphasized that the velocity fields used in our model have a rather simple, idealized structure. More realistic fields can be expected to include significant $m=1$ and $m>2$-contributions and have a more complex topology. Since we made linear calculations with time-independent velocities, it is beyond the scope of this study whether an interaction lasting roughly 1 Gyr $\left(9 \times 10^{8}\right.$ yr in the case of M 81 according to the simulation of Thomasson \& Donner 1993) will generate significant nonaxisymmetric magnetic fields. For realistic values of $\alpha_{0}$ we found typical growth times of the $m=1$ modes of a few $10^{8} \mathrm{yr}$, so it seems plausible that an interaction can produce the observed field configurations. They would also last for approximately the same time after an encounter.

Interestingly, mode coupling due to nonaxisymmetric velocity components plays only a minor role in our model. A direct excitation of $m=1$ magnetic field modes by means of $\boldsymbol{u}_{\mathbf{2}} \times \boldsymbol{B}_{\mathbf{1}}$ type interactions, as observed by Moss (1995, 1996), was not found. However, a direct comparison of the results is difficult since the model of Moss does not discriminate between modes even and odd with respect to the equator.

\section{References}

Beck, R., Brandenburg, A., Moss, D., Shukurov, A., \& Sokoloff, D. D. 1996, ARA\&A, 34, 155

Berkhuijsen, E. M., Horellou, C., Krause, M., et al. 1997, A\&A, 318,700

Buczilowski, U. R., \& Beck, R. 1991, A\&A, 241, 47

Deinzer, W., Grosser, H., \& Schmitt, D. 1993, A\&A, 273, 405

Elstner, D., Meinel, R., \& Beck, R. 1992, A\&AS, 94, 587

Elstner, D., Meinel, R., \& Rüdiger, G. 1990, Geophys. Astrophys. Fluid Dyn., 50, 85

Elstner, D., Rüdiger, G., \& Schultz, M. 1996, A\&A, 306, 740

Ferrière, K. M., \& Schmitt, D. 2000, A\&A, 358, 125

Hoyng, P., Schmitt, D., \& Teuben, L. J. W. 1994, A\&A, 289, 265

Hummel, E., \& Beck, R. 1995, A\&A, 303, 691

Krause, M., Beck, R., \& Hummel, E. 1989, A\&A, 217, 17

Moss, D. 1995, MNRAS, 275, 191

Moss, D. 1996, A\&A, 315, 63

Moss, D., Brandenburg, A., Donner, K. J., \& Thomasson, M. 1993, ApJ, 409, 179

Moss, D., Shukurov, A., \& Sokoloff, D. 2000, A\&A, 358, 1142

Parker, E. N. 1971a, ApJ, 163, 255

Parker, E. N. 1971b, ApJ, 164, 491

Poezd, A., Shukurov, A., \& Sokoloff, D. 1993, MNRAS, 264, 285

Rädler, K.-H. 1986, in Plasma Physics, ESA SP-251, 569

Rohde, R., Beck, R., \& Elstner, D. 1999, A\&A, 350, 423

Rüdiger, G., \& Kichatinov, L. L. 1993, A\&A, 269, 581

Schmitt, D., \& Schüssler, M. 1989, A\&A, 223, 343

Schreiber, E., \& Schmitt, D. 2000, A\&A, submitted

Schultz, M., Elstner, D., \& Rüdiger, G. 1994, A\&A, 286, 72

Thomasson, M., \& Donner, K. J. 1993, A\&A, 272, 153

Torkelsson, U., \& Brandenburg, A. 1994, A\&A, 292, 341 